\title{
Early cytokine signatures of ischemia/ reperfusion injury in human orthotopic liver transplantation
}

Rebecca A. Sosa, ${ }^{1}$ Ali Zarrinpar, ${ }^{2}$ Maura Rossetti,, Charles R. Lassman, ${ }^{1}$ Bita V. Naini, ${ }^{1}$ Nakul Datta, ${ }^{2}$ Ping Rao, ${ }^{1}$ Nicholas Harre, ${ }^{1}$ Ying Zheng, ${ }^{1}$ Roberto Spreafico, ${ }^{3,4}$ Alexander Hoffmann, ${ }^{3,4}$ Ronald W. Busuttil, ${ }^{2}$ David W. Gjertson, ${ }^{1}$ Yuan Zhai, ${ }^{2}$ Jerzy W. Kupiec-Weglinski, ${ }^{1,2}$ and Elaine F. Reed ${ }^{1}$

'Department of Pathology and Laboratory Medicine, '2Department of Surgery, ${ }^{3}$ Department of Microbiology, Immunology, and Molecular Genetics, and ${ }^{4}$ Institute for Quantitative and Computational Biosciences, UCLA, California, USA.

BACKGROUND. Orthotopic liver transplant (OLT) is the primary therapy for end-stage liver disease and acute liver failure. However, ischemia/reperfusion injury (IRI) can severely compromise allograft survival. To understand the evolution of immune responses underlying OLT-IRI, we evaluated longitudinal cytokine expression profiles from adult OLT recipients before transplant through 1 month after transplant.

METHODS. We measured the expression of 38 cytokines, chemokines, and growth factors in preoperative and postoperative recipient circulating systemic blood (before transplant and 1 day, 1 week, and 1 month after transplant) and intraoperative portal blood (before and after reperfusion) of 53 OLT patients and analyzed this expression in relation to biopsy-proven IRI ( $n=26$ IRI+; 27 IRI-), clinical liver function tests early (days 1-7) after transplant, and expression of genes encoding cytokine receptors in biopsies of donor allograft taken before and after reperfusion.

RESULTS. Bilirubin and arginine transaminase levels early after transplant correlated with IRI. Fourteen cytokines were significantly increased in the systemic and/or portal blood of IRI+ recipients that shifted from innate to adaptive-immune responses over time. Additionally, expression of cognate receptors for 10 of these cytokines was detected in donor organ biopsies by RNAseq.

CONCLUSION. These results provide a mechanistic roadmap of the early immunological events both before and after IRI and suggest several candidates for patient stratification, monitoring, and treatment.

FUNDING. Ruth L. Kirschstein National Research Service Award T32CA009120, Keck Foundation award 986722, and a Quantitative \& Computational Biosciences Collaboratory Postdoctoral Fellowship.

Conflict of interest: The authors have declared that no conflict of interest exists.

Submitted: July 21, 2016 Accepted: October 25, 2016 Published: December 8, 2016

Reference information: JCI Insight. 2016;1(20):e89679. doi:10.1172/jci.insight.89679.

\section{Introduction}

Orthotopic liver transplant (OLT) is the primary therapy for end-stage liver disease and acute liver failure. Ischemia/reperfusion injury (IRI) occurs as an inevitable consequence of the transplant process, beginning with organ procurement and preservation and followed by reperfusion of the donor organ with recipient blood during transplant (1). Data from murine models have indicated that liver IRI has hypoxic cellular stress and inflammation-mediated injury components (2-5). Local circulatory damage first induces endogenous reactive oxygen species production causing hepatocyte death. This cellular damage initiates the second phase by recruiting and activating innate immune cells at the site of injury. IRI is then further exacerbated by the adaptive immune system; indeed, activated CD4 ${ }^{+} \mathrm{T}$ cells are essential in promoting IRI-related inflammation and hepatocyte damage in mice. IRI can lead to primary graft nonfunction and need for retransplantation (6) and predisposes the recipient to both acute and chronic rejection and graft loss as well as decreases the pool of transplantable organs. Although IRI is a signifi- 
cant clinical problem across all solid organ transplants, remarkably few studies have been conducted in the setting of human transplantation to understand its mechanistic underpinnings.

Several clinical tests are routinely used to monitor liver dysfunction (7). These include increased elevated blood levels of the intracellular liver enzymes alanine transaminase (ALT) and arginine transaminase (AST), which are released upon hepatocellular damage. Total bilirubin is also used as a measure of liver function, as it indicates either impaired heme catabolism or cholestasis, a partial to complete blockage of bile flow. Finally, prothrombin time, reported as the international normalized ratio (INR), is a common blood clotting test used as a measure of liver biosynthetic function. However, all of these tests suffer from poor sensitivity and specificity, and it is uncertain how these tests relate to IRI, which is currently only identifiable by biopsy.

The liver is home to a tightly regulated cytokine network. Hepatocytes are highly susceptible to cytokine activity in physiological and pathophysiological conditions, both acute and chronic (8). In the adult liver, approximately $30 \%$ of the liver's cells are nonhepatocytes and include hepatic stellate cells, liver sinusoidal endothelial cells, macrophages (Kupffer cells), dendritic cells, and lymphocytes, which can produce a variety of cytokines, chemokines, and growth factors acting systemically or in a paracrine manner on hepatocytes and nonparenchymal cells $(9,10)$. Additionally, several cytokines are key mediators of the hepatic acute phase response (11). Any of these cytokines might be induced upon acute liver injury, such as IRI; however, their involvement and kinetics in this process remain unclear.

Here, we characterized the evolution of the immune response in 53 OLT recipients using multiplex cytokine profiling of recipient circulating systemic and portal venous blood before, during, and after OLT (up to 1 month after transplant). In addition, we analyzed clinical liver function tests early after transplantation and correlated gene expression of cytokine receptors in allograft biopsies obtained before and after reperfusion. We show that patients classified as either IRI+ $(n=26)$ or IRI- $(n=27)$ by histopathology have distinct cytokine signatures at each time point and display differential levels of bilirubin and AST shortly after transplant. We further show a switch from innate to adaptive immune responses in IRI+ patients that appears to be driven by cytokines from within the allograft itself. Finally, we demonstrate the expression of genes encoding cognate receptors for many of the increased cytokines found in IRI+ patients. Taken together, these results point to key differences between IRI+ and IRI- patients' immune statuses before, during, and after transplant that could lead to a chronic state of inflammation, rather than a complete resolution of an acute response. Understanding the timing of immune events, and the contribution of both the donor and recipient to the inflammatory milieu, is critical to developing much needed therapeutic strategies to reduce IRI and improve OLT outcomes.

\section{Results}

OLT recipient and donor characteristics. To test the hypothesis that cytokine and chemokine profiles can be used to assess the immune status of the OLT recipient and the risk of IRI, we analyzed plasma before and after transplant and liver allograft biopsies after reperfusion from 53 recipients enrolled in our IRB-approved study after written, informed consent was obtained. To determine the incidence of IRI in our study population, intraoperative biopsies of the liver allograft after reperfusion were assessed according to a modified Suzuki (12) histological grading system (Table 1). Our scoring criteria consisted of presence of neutrophilic inflammatory infiltrates along with necrotic hepatocytes; patients scoring a 0 or 1 overall were designated as IRI-, and patients scoring a 2-4 overall were designated as IRI+.

All 53 recipients were scored for IRI severity by histopathology and 26 were graded as IRI+ and 27 were graded as IRI-. Plasma samples from circulating systemic blood were collected preoperatively (PO) and at 1 day (D1), 1 week (W1), and 1 month (M1) after transplant. Intraoperative blood was also collected from the portal vein (PV) of the recipient before and after it was used to reperfuse the donor liver (liver flush $[\mathrm{LF}])$. The demographic data and clinical parameters of the recipients and donors are shown in Table 2. Multivariate analysis did not find any correlation between degree of IRI and any of the recipient or donor demographics or clinical parameters, including major factors previously implicated in IRI, such as age (13), gender (14), MELD score $(15,16)$, or extended cold ischemia time (17).

Clinical liver function tests can be informative regarding IRI status. Transplant recipients were evaluated for liver function with standard-of-care tests, including AST, ALT, serum total bilirubin, and INR. Measurements were obtained daily during the first week after transplant (Figure 1). Bilirubin was statistically higher in IRI+ recipients than in IRI- recipients on day 2 and 3 after transplant. Both ALT and AST showed a downward trend over time in both IRI+ and IRI- recipients. However, AST values 
Table 1. Histopathological grading system of liver IRI in human OLT

\begin{tabular}{|c|c|c|c|c|c|c|}
\hline Feature & Description & \multicolumn{5}{|c|}{ Grade $^{A}$} \\
\hline \multicolumn{7}{|l|}{$\begin{array}{l}\text { Histopathological } \\
\text { features evaluated } \\
\text { as part of IRI grading } \\
\text { system }\end{array}$} \\
\hline Inflammation & $\begin{array}{l}\text { Neutrophilic } \\
\text { infiltrates }\end{array}$ & None & $\begin{array}{l}\text { Scattered neutrophils in } \\
\text { sinusoids } \pm \text { rare clusters of } \\
\text { neutrophils }\end{array}$ & $\begin{array}{l}\text { Sinusoidal } \\
\text { neutrophils }+ \\
\text { small clusters of } \\
\text { neutrophils in most } \\
\text { lobules }\end{array}$ & $\begin{array}{l}\text { Sinusoidal } \\
\text { neutrophils in } \\
\text { all lobules and } \\
\text { prominent clusters } \\
\text { of neutrophils in } \\
>50 \% \text { of lobules }\end{array}$ & $\begin{array}{c}\text { Pan-lobular } \\
\text { sinusoidal } \\
\text { neutrophils in all } \\
\text { lobules }\end{array}$ \\
\hline Necrosis & $\begin{array}{l}\text { Lobules with } \\
\text { necrotic clusters }\end{array}$ & None & $\begin{array}{l}\text { Single-cell necrosis in one or } \\
\text { more lobule }\end{array}$ & $\begin{array}{l}\text { At least } 1 \text { cluster } \\
\text { of necrotic cells } \pm \\
\text { single cell necrosis }\end{array}$ & $\begin{array}{c}\text { Clusters of necrotic } \\
\text { cells in most } \\
\text { lobules, at least one } \\
\text { with more than } 10 \\
\text { necrotic cells per } \\
\text { cluster }\end{array}$ & $\begin{array}{l}60 \% \text { of parenchyma } \\
\text { is necrotic or there is } \\
\text { e pan-lobular necrosis } \\
\text { in more than one } \\
\text { lobule }\end{array}$ \\
\hline
\end{tabular}

Additional

histopathological

features evaluated

Congestion

Sinusoidal

None Scattered sinusoids or Zone 3 congestion in

Zone 2 and 3

Pan-lobular prominent zone 3 sinusoidal most lobules

congestion in most lobules

gestion in most

Ballooning

Hepatocellular

Scattered ballooning Prominent clusters Pan-lobular cells in most lobules of ballooning cells in hepatocellular and or prominent most lobules ballooning involving cluster in 1 lobule more than one Iobule

\begin{tabular}{|c|c|c|c|c|c|c|}
\hline $\begin{array}{l}\text { Large-droplet } \\
\text { macrovesicular steatosis } \\
\text { (single/few large lipid } \\
\text { vacuoles occupying } \\
>50 \% \text { of a cell with } \\
\text { nuclear displacement) }\end{array}$ & $\begin{array}{l}\text { \% parenchymal } \\
\text { replacement }\end{array}$ & none & $\leq 10 \%$ & $10-20 \%$ & $20 \%-30 \%$ & $\geq 30 \%$ \\
\hline Cholestasis & $\begin{array}{l}\text { Canalicular or } \\
\text { hepatocellular }\end{array}$ & None & Rare zone 3 & $\begin{array}{l}\text { Prominent in } \geq 1 \\
\text { lobule }\end{array}$ & $\begin{array}{l}\text { Prominent most } \\
\text { lobules }\end{array}$ & $\begin{array}{l}\text { Prominent zone } 3 \text { in } \\
\text { most lobules, zone } 1\end{array}$ \\
\hline
\end{tabular}
$\geq 1$ lobule

${ }^{A} 1$, minimal; 2 , mild; 3 , moderate; 4 , severe.

were significantly increased in IRI+ recipients on days 5 and 6 after transplant as compared with IRIrecipients. ALT and INR values were comparable in the two groups at time points tested. These data suggest that disturbances in bilirubin levels early in the first week after transplant and AST later that same week are mediated by IRI.

Circulating cytokines and chemokines are differentially expressed in IRI+ and IRI-patients before and after OLT. We used unsupervised hierarchical clustering to first identify patterns before and after transplant of 38 cytokines, chemokines, and growth factors in the systemic blood (Figure 2). The algorithm clustered the samples by similarity of cytokine profiles. Remarkably, the overall cytokine pattern in peripheral blood segregated patients by IRI status, except for samples collected at M1, suggesting that IRI induces a distinct cytokine signature very early after transplant.

The algorithm clustered analytes into 3 main groups. The first cluster contained 3 Th2-type or antiinflammatory cytokines (IL-13, IL-10, and IL-1Ra), no chemokines, and 3 growth factors (IL-15, IL-2, 


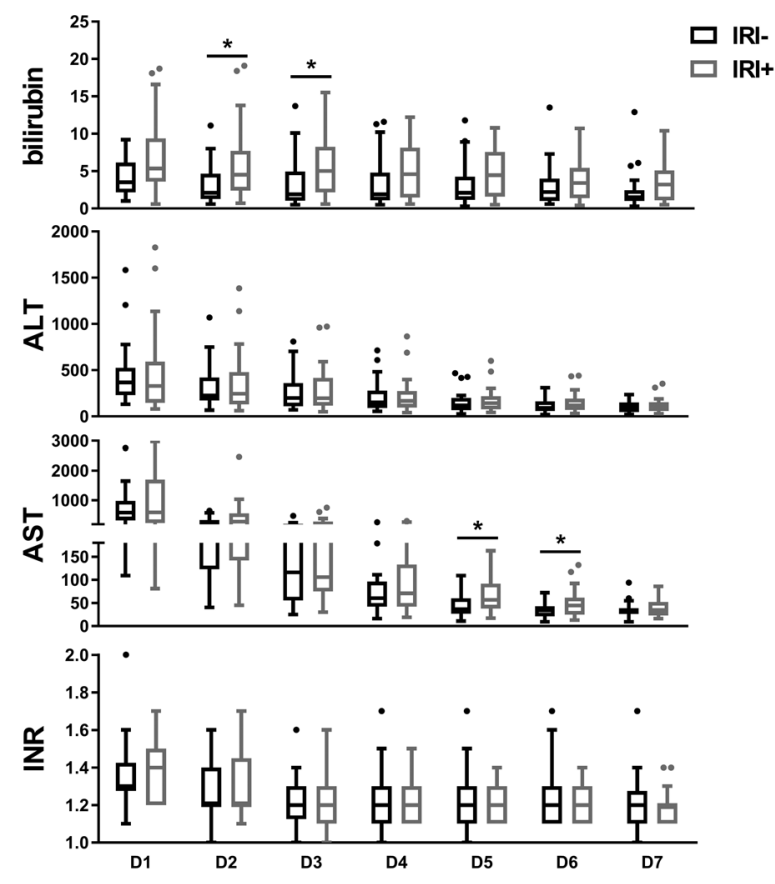

Figure 1. Recipient clinical tests of liver function after OLT-IRI correlate with IRI. Four clinical liver function tests were performed on recipient circulating peripheral blood taken on days 1-7 after transplant (D1-D7). Data are presented as Tukey boxand-whisker plots: whiskers are inner fences reaching 1.5 times the interquartile range and boxes represent the interquartile ranges, dots indicate outlying values, and lines represent median values for each day: bilirubin $(\mathrm{mg} / \mathrm{ml})$, ALT and AST (U/I), or INR. $n=53 ; 26 \mathrm{IRI}$ - and $27 \mathrm{IRI}+.{ }^{*} P<0.05$. The Wilcoxon rank-sum test was used for comparison between IRI+ and IRI- patient groups.

and G-CSF). The median for this group of cytokines was highest in IRI+ patients at D1. The second cluster contained 8 cytokines (IL-12p70, IL-5, IL-1 $\beta$, IL-17A, TNF- $\alpha$, IL-9, sCD40L, and IFN- $\alpha 2), 6$ chemokines (CX3CL1, CCL4, CCL7, CXCL1, CCL2, and CCL11), and 4 growth factors (Flt-3L, VEGF, FGF-2, and IL-3). Overall, the median for these analytes was higher in the IRI+ group compared with the IRI- group PO, at D1, and at W1. In addition, the growth factor Flt-3L showed strongest expression at M1 for both groups of patients as compared with any other time point, with expression in the IRI+ group being higher than that in the IRI- group. The third cluster was enriched in analytes with proinflammatory activity and included 6 cytokines (TNF- $\beta$, IL-12p40, IL-4, IL-6, IFN- $\gamma$, and IL-1 $\alpha$ ), 4 chemokines (CCL22, CCL3, CXCL8, and CXCL10), and 4 growth factors (IL-7, EGF, GM-CSF, and TGF- $\alpha$ ). The median for this group was higher overall in IRI+ patients compared with IRI- patients at $\mathrm{PO}$, suggesting predisposition to IRI damage.

We then evaluated each cytokine individually for its correlation with IRI outcome (Figure 3). PO blood from IRI+ recipients showed significantly increased expression of the cytokines IL-1Ra, TNF- $\alpha$, IL-5, and IL-13; the chemokine CXCL8; and the growth factors IL-2, IL-7, and EGF compared with IRI- recipients. Early after transplantation (D1), the cytokines IL-1Ra, IL-4, IL-13, and IL-17A were significantly higher in IRI+ patients compared with IRI- patients. W1 following transplantation, the cytokines IL- $1 \alpha$ and IL-4 were significantly higher in the circulation of IRI+ patients, as was the chemokine CCL11 and the growth factors IL-2, IL-3, and IL-7. Late after IRI (M1), significant increases in IL-1Ra were seen in IRI+ patients and IL-1 $\alpha$ was no longer significantly different between IRI groups. At this time point, IL-13 was significantly increased in IRI+ patients. IL-17A was significantly increased in IRI+ patients at M1, as were IL-2 and IL-3 (Figure 3). Other cytokines tested did not show significant differences at these time points between IRI groups (Supplemental Figure 1). These data show a clear switch from primarily acute/innate inflammatory mediators to chronic/adaptive inflammatory mediators, occurring by D1 after transplant, that persists in IRI+ patients up through at least M1 after transplant.

Donors and recipients contribute differentially to cytokine expression during OLT. OLT-IRI severity could potentially be affected by mediators from either, or both, of the donor and recipient immune systems. Therefore, we explored the contributions of cytokines, chemokines, and/or growth factors from these distinct sources by comparing recipients' portal venous blood before (PV) or after being flushed through the donor organ (LF) after it has been anastomosed, which constitutes the actual reperfusion event (Figures 4 and 5). PV blood has not yet come in contact with the donor allograft and, therefore, serves as a baseline for the recipient's soluble immune contribution to IRI at the time of reperfusion. We reason that the LF sample should still contain the same factors as the PV sample but will also contain soluble factors released from the allograft during the reperfusion event.

For the portal blood samples taken before and after reperfusion, the algorithm clustered the samples by origin (PV or LF) rather than IRI status, indicating that the transplanted liver is contributing or retaining cytokines selectively, modifying the overall cytokine signature of the portal blood (Figure 4). Cytokines clustered into 5 main groups. Of note, IL-1Ra clustered by itself and was highly enriched after portal blood was flushed through the liver, especially in IRI+ patients. The second cluster contained 4 cytokines (IL-13, IL-6, IL-5, and IL-9), 2 chemokines (CXCL1 and CX3CL1), and 5 growth factors (IL-15, FGF-2, IL-3, Flt-3L, and IL-7). This group showed their strongest expression in the portal blood of IRI+ patients, regardless of whether the sample was obtained before (PV) or after reperfusion (LF). The third cluster contained 3 cytokines (IL-1 $\beta$, IL-12p70, and LT- $\alpha$ ), 1 chemokine (CXCL8), and 1 growth factor (IL-2). 
This group showed high expression in the portal blood samples of IRI+ patients taken prior to reperfusion through the liver (PV). The fourth cluster was the largest, with 6 proinflammatory, Th1/17-type cytokines (IFN- $\gamma$, TNF- $\alpha$, IL-1 $\alpha$, IFN- $\alpha 2$, IL-12p40, and IL-17A), 6 chemokines (CCL3, CXCL10, CCL7, CCL11, CCL7, and CCL2), and 3 growth factors (TGF- $\alpha$, GM-CSF, and VEGF). Similar to the second cluster, this group had higher cytokine expression in portal blood taken prior to reperfusion from OLT recipients. The fifth cluster contained 3 cytokines (sCD40L, IL-4, and IL-10), 1 chemokine (CCL22), and 2 growth factors (EGF and G-CSF); this group had higher expression in PV, especially in IRI- patients.

We then analyzed each cytokine individually, similarly to what was done for the systemic blood. PV blood from IRI+ patients showed significant increases in the chemokine CXCL8 and the growth factor IL-3 over PV blood from IRI- patients (Figure 5). CXCL8 and IL-3 were also found in LF. In addition, LF from IRI+ patients also showed significant increases over IRI- patients in the expression of the cytokines IL-1Ra and IL-12p70 and the growth factor IL-2, which were not seen in PV blood samples. Recipient systemic blood taken before operation showed increases in both IL-1Ra and IL-2 as well (Figure 3); however, IL-12p70 was only significantly increased in IRI+ patient's LF samples (Figure 5), implicating the donor organ as the significant source of this adaptive cytokine. Other cytokines tested did not show significant differences (Supplemental Figure 2).

Genes encoding cognate receptors for IRI-related recipient cytokines are expressed in donor livers. Most cytokines signal through cognate receptors expressed on the surface of responsive cells. As such, we examined biopsy samples before and after reperfusion from a smaller representative cohort of IRI+ $(n=4)$ and IRI- $(n=6)$ patients for the expression of genes encoding receptors for any of the 14 cytokines that were found to be upregulated in IRI+ patient blood at any time point (Figure 6). We found expression of IL-1R1, whose protein product can bind IL-1 $\alpha$, IL-1 $\beta$, or IL-1Ra, as well as expression of the gene that encodes the decoy receptor for these cytokines, IL-1R2. Additionally, we found intragraft expression of both TNFSR1A and TNFSR1B, which encode the receptors TNFR1 and TNFR2, respectively, both of which can bind the cytokine TNF- $\alpha$. The genes IL-4R, IL-13RA1, and IL-13RA2 were all found to be expressed. These genes encode the receptors for the cytokines IL-4 (IL-4R and IL-13RA1) and/or IL-13 (IL-13RA1 and IL-13RA2). We found expression of IL-17RA and IL-17RC, which encode receptors for IL-17A; CXCR1 and CXCR2 genes, which encode receptors for the chemokine CXCL8; and CCR2 and CCR5, which encode receptors for CCL11. IL2RA and IL2RB, which encode cognate receptors for IL-2, were also present, as was EGFR, which encodes the receptors for EGF. We did not see significant changes in the expression levels of any of these cytokine receptor-encoding genes based on either the reperfusion event or patient IRI status, suggesting that the IRI risk factor is at the cytokine level rather than the receptor level. No genes encoding any of the known potential receptors for IL-3, IL-5, IL-7, or IL-12 were detectable in biopsy tissues, suggesting that the liver might not be responsive to these cytokines.

Histopathological grading of IRI in human OLT is based on inflammation and necrosis, with additional biopsy features evaluated. Although our principal scoring criteria consisted of presence of neutrophilic inflammatory infiltrates along with necrotic hepatocytes, IRI+ patients typically presented one or more additional histopathological features, including sinusoidal congestion, hepatocyte ballooning, macrovesicular steatosis, and cholestasis (Figure 7A). Figure 7A shows examples of biopsies from 4 representative OLTIRI patients, with marked presence of these additional features along with substantial inflammation and necrosis. No specific combination of these features was noted among IRI patients with them; however, over two-thirds (18 of $26,69 \%$ ) of IRI+ patients presented with at least one other prominent histopathological feature in addition to the well-accepted measures of IRI (inflammation and necrosis), with $31 \%$ (8 of 26) also having an increase in one other feature, $27 \%$ (7 of 26) presenting with two additional features, and $12 \%$ (3 of 26) with three other features (Figure 7B). Nearly half (44\%) of IRI- patients had no increase in any of the 4 extrahistopathological features. $22 \%$ of IRI-patients had an increase in only one additional feature evaluated, and $26 \%$ had an increase in two of the histopathological features. No IRIpatients had increases in three or four of the additional histopathological features scored.

Sinusoidal congestion has been associated with long-term liver dysfunction (18). This congestion is typically due to cardiac pump dysfunction, which causes a similar pulse of prolonged ischemia, followed by relatively sudden reperfusion in the liver as occurs during OLT. About half of IRI+ patients had increased amounts of congestion ( 12 of $26=46 \%$ ), whereas only $22 \%$ ( 6 of 27 ) of IRI- patients had some signs of congestion (Figure 7C). We evaluated ballooning degeneration as an early form of hepatocyte death via apoptosis. $27 \%$ (7 of 26) of IRI+ patients presented with ballooning, which was similar to that seen in 


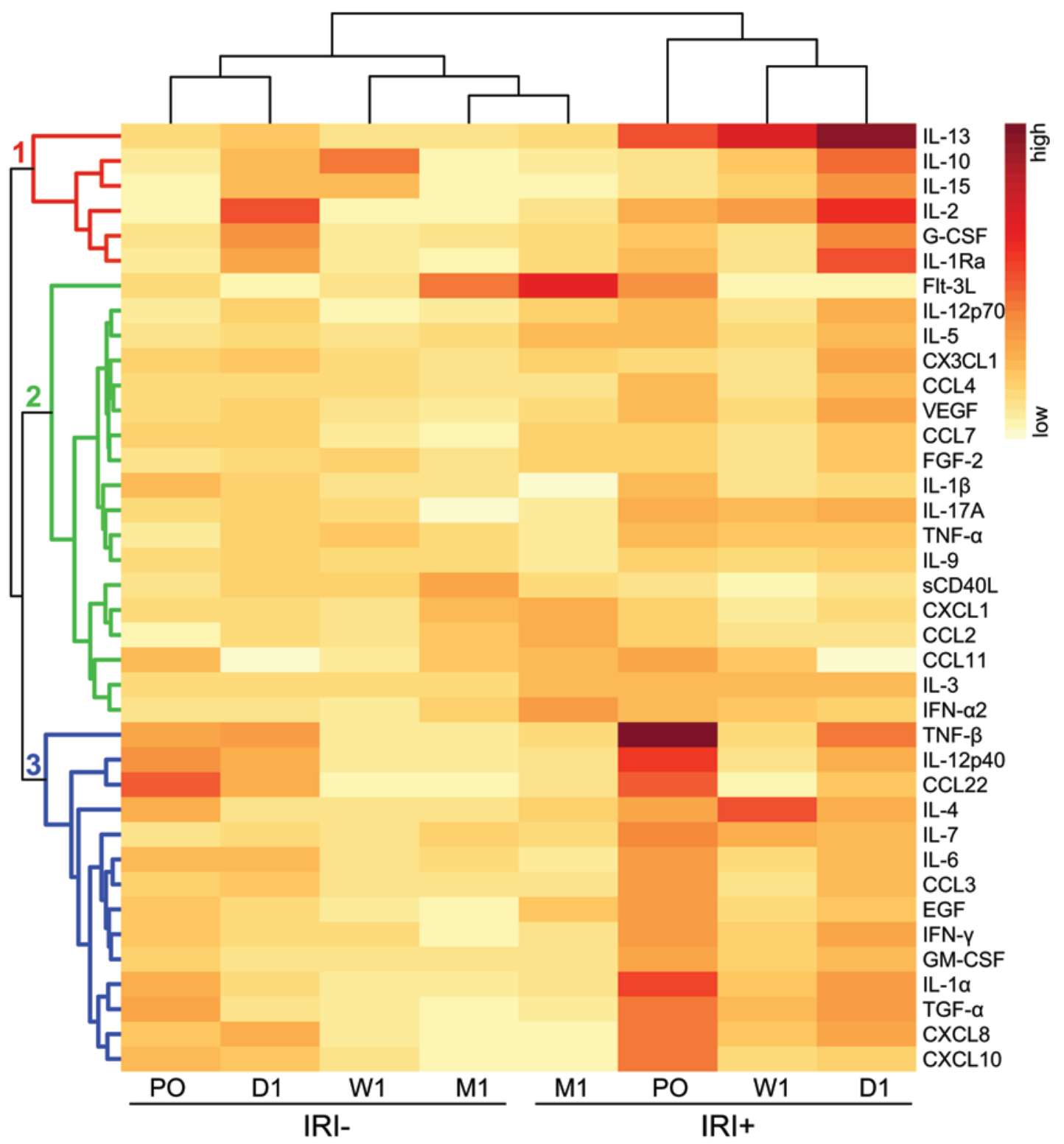

Figure 2. Recipient circulating cytokines, chemokines, and growth factors cluster by IRI status and then time point relative to OLT. Thirty-eight cytokines, chemokines, and growth factors were measured in circulating blood of OLT recipients preoperatively (PO) and at postoperative day 1 (D1), week 1 (W1), or month 1 (M1) time points by Luminex multiplex assay. Shown is a heatmap in which the rows represent cytokines, the columns represent IRI status at each time point, and the colors represent normalized median cytokine concentration values (white $=$ low, red = high). The rows and columns are ordered based on the results of unsupervised hierarchical clustering, with dendrograms for the cytokines and patient groups shown on the vertical and horizontal axes, respectively.

IRI- patients ( 7 of $27=26 \%$ ). Large-droplet macrovesicular steatosis, when accompanied with prominent inflammation, has been shown to impair liver regeneration, due to increased hepatocellular lipid peroxidation and damage (19). In IRI+ patients, $10 \%$ of parenchymal hepatocytes consistently had large-droplet macrovesicular steatosis, while this was not the case in IRI- patients. 38\% (10 of 26) of IRI+ patients had multiple zone 3 large-droplet macrovesicular steatosis in most lobules, while only 19\% (5 of 27) of IRIpatients had increased steatosis (Figure 7C). Finally, we evaluated cholestasis. We detected only rare zone 3 canalicular or hepatocellular cholestasis, suggesting that the presence or absence of cholestasis is more important than the degree of cholestasis at this early intraoperative time point (Figure 7C).

To determine if additional features seen by histopathology were relevant in assessing IRI severity and outcome, we evaluated our clinical liver function data from the first week following OLT. AST at days 5 and 6 was elevated in IRI+ patients with one or more additional histopathological features (Figure 7D) as com- 
Table 2. OLT patient and donor demographics

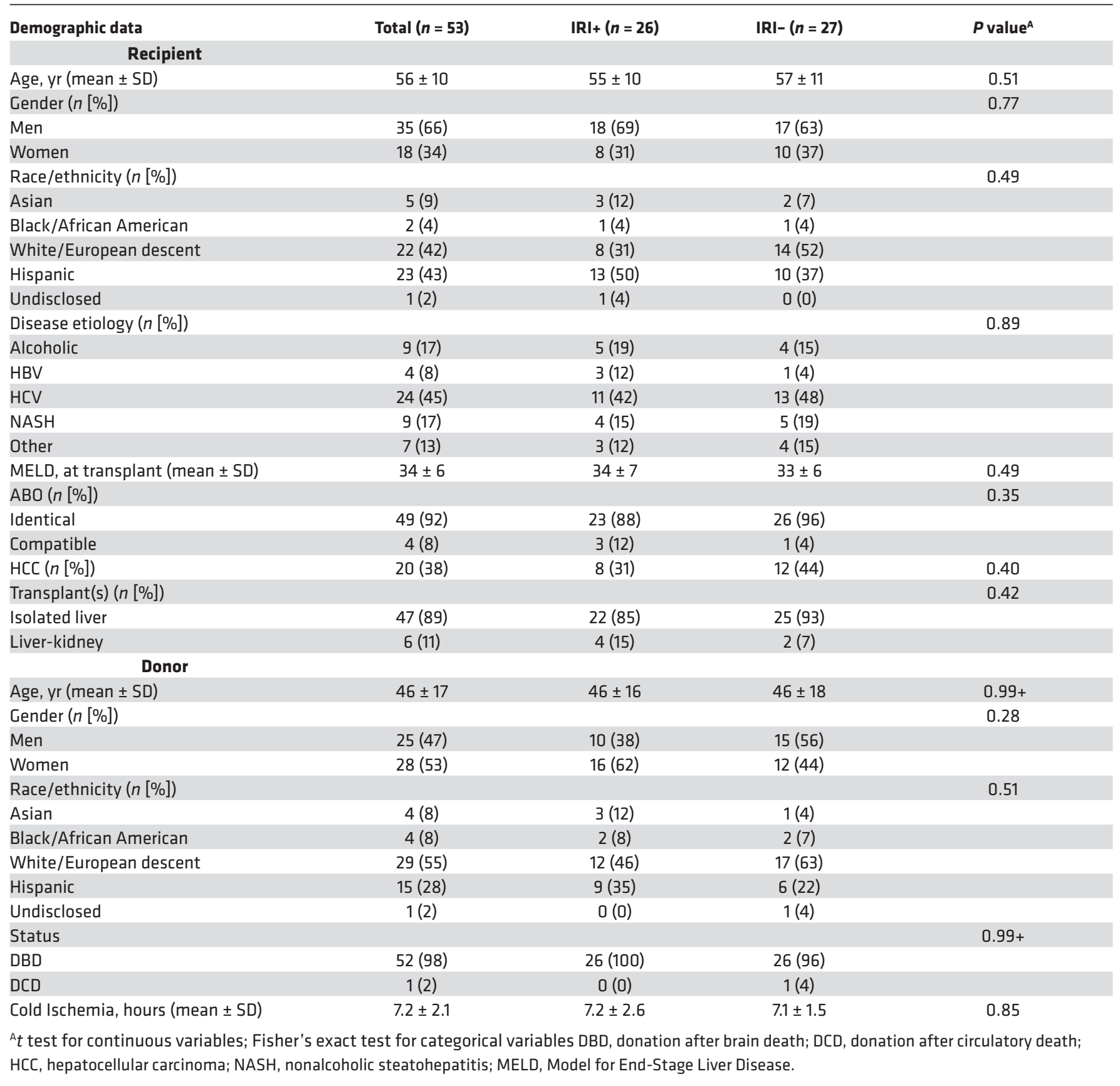

pared with IRI- patients with one or more additional features or IRI+ or IRI- patients with no additional histopathological features. These data suggest that the presence of additional histological features correlates with increased liver dysfunction shortly after transplant in IRI+ patients. Additionally, in evaluating the outcomes of our patient cohort within the first year following transplant (Table 3), we found that OLT recipients designated as IRI+ based on our scoring system were more likely to suffer complications requiring more than one "for-cause" biopsy (50\% IRI+ vs. 11\% IRI-), showed signs of more severe complications such as acute cellular rejection (ACR) (33\% IRI+ vs. $0 \%$ IRI-) or antibody-mediated rejection (AMR) (33\% IRI+ vs. $11 \%$ IRI-) versus those patients designated as IRI- who mostly had HCV recurrence (67\% IRI- vs. $17 \%$ IRI+), and showed signs of more than one complication ( $67 \%$ IRI+ vs. $0 \%$ IRI-). 

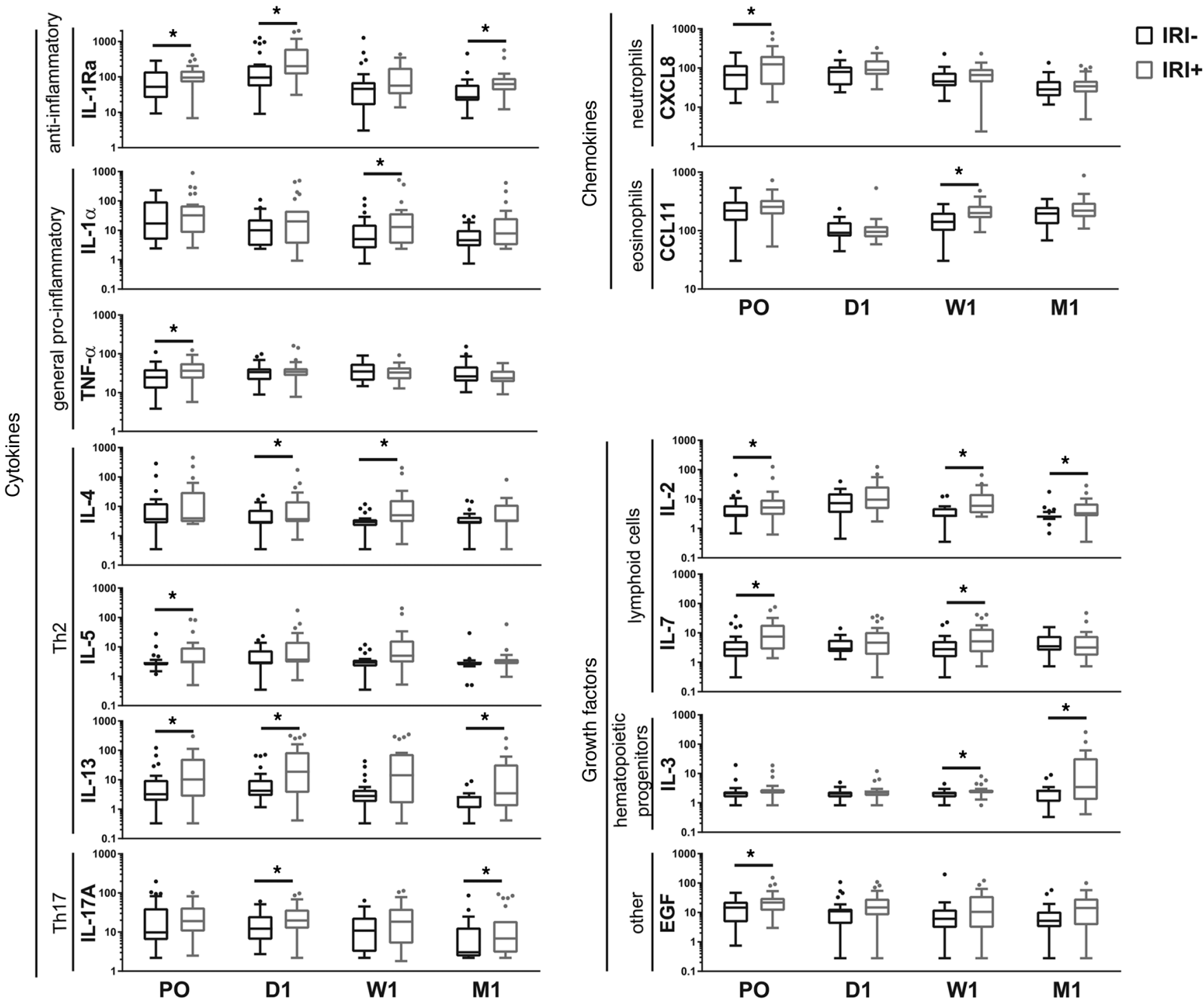

Figure 3. Recipient circulating cytokines, chemokines, and growth factors are altered before and after OLT. Of 38 cytokines, chemokines, and growth factors tested by Luminex Multiplex assay, 13 were significantly higher in IRI+ patients versus IRI- patients either preoperatively (PO) or at postoperative day 1 (D1), week 1 (W1), or month 1 (M1) time points. Data are presented as Tukey box-and-whisker plots: whiskers are inner fences reaching 1.5 times the interquartile range and boxes represent the interquartile ranges, dots indicate outlying values, and lines represent median values for each time point. $n=$ 53; $26 \mathrm{IRI}$ - and $27 \mathrm{IRI}+{ }^{*} P<0.05$. The Wilcoxon rank-sum test was used for comparison between IRI+ and IRI- patient groups.

\section{Discussion}

Severe IRI occurs at several key time points during the process of OLT, starting with oxidative stress in the organ donor, continuing during cold storage and transport of the organ, and again when the organ is warmed and reperfused with blood from the recipient. These events all contribute to the overall degree of IRI and provide a plethora of signals to and from both the donor and the recipient's immune systems that ultimately result in immune cell recruitment and activation to the site of injury. Therefore, defining the qualitative and quantitative immune events of IRI in human OLT is critical to the development of targeted therapies as well as improvement of overall OLT outcome. Here, we examined the evolution of the immune response in OLT recipients in the context of IRI, with the overall goal of determining cytokine and chemokine profiles that could potentially be used to assess the immune status of the OLT recipient and risk of IRI. We have characterized the relationship between expression of cytokines, chemokines, and growth factors and clinical graft function to biopsy-proven IRI. We have shown that IRI+ patients display dysregulation in liver function represented by increased bilirubin and AST levels shortly after transplant. 


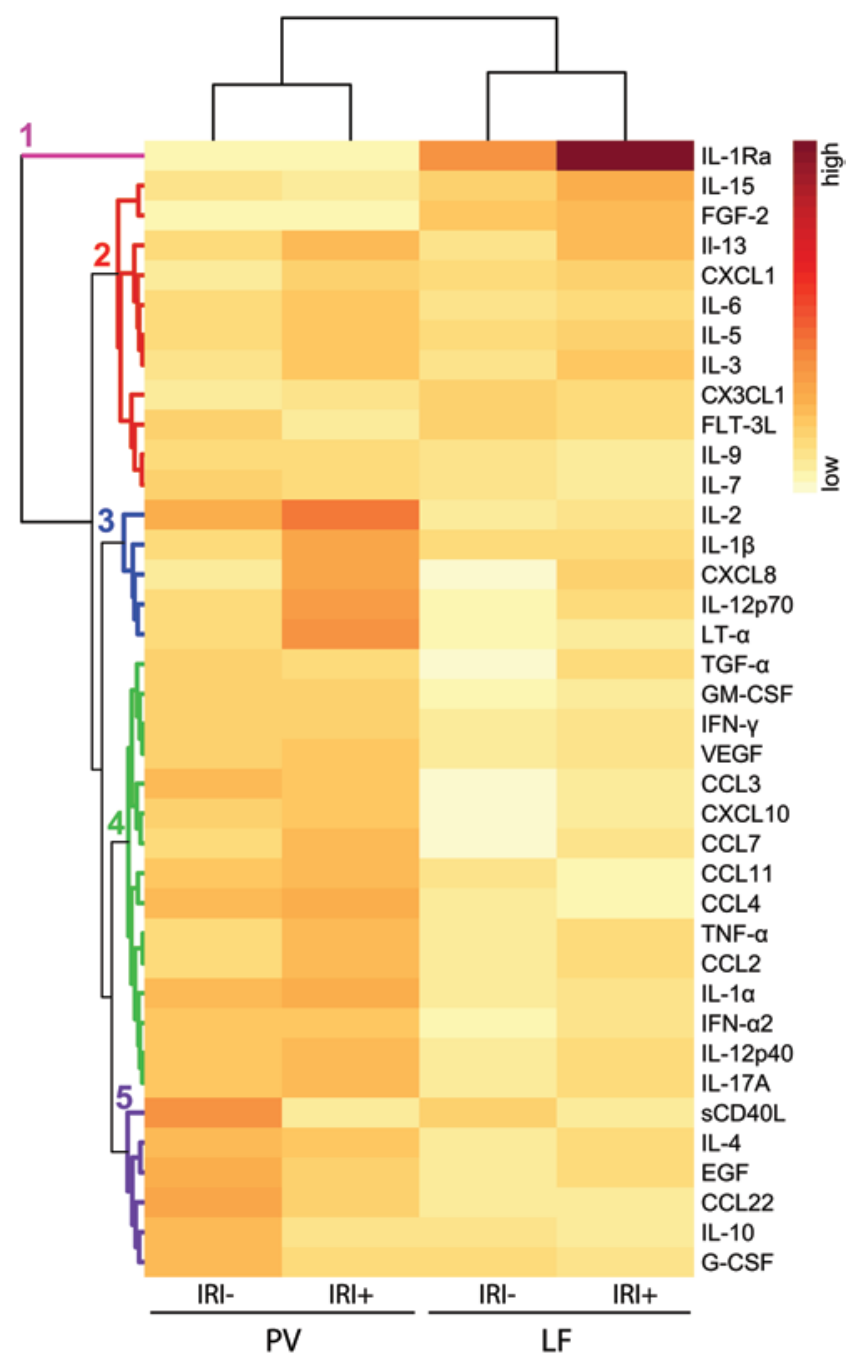

Notably, our study shows that IRI+ patients have a distinct cytokine signature compared with IRI- patients already at the time of transplant surgery (PO), suggesting a specific immune phenotype that might predispose an OLT recipient to IRI. This signature comprises elevated levels of TNF- $\alpha$, IL-5, IL-13, IL-2, CXCL8, IL-7, $\mathrm{IL}-1 \mathrm{Ra}$, and EGF. Any individual cytokine or combination of these cytokines might be related to the underlying disease for which patients are necessitating OLT, including viral hepatitis or hepatocellular injury due to nonalcoholic steatohepatitis. However, we did not see any relationship between any specific etiology and these cytokines' expression within our patient cohort. Alternatively, these cytokines could potentially be related to patient prior immune history, such as prior infections, allergies, autoimmunity, cancers, and/or other sterile inflammatory events, which may be unaccounted for in their records and therefore unable to be assessed. Regardless of their origin, the increased expression of these cytokines in the circulating systemic blood of the OLT recipient is positively associated with IRI risk. No association was found between increased expression of cytokines and severity of IR damage or donor factors, including extended cold ischemia times, steatosis or donation after circulatory death status, or first year after transplant complications requiring a "for-cause" biopsy, which may be due to the limited number of data points for these variables in our cohort (Tables 2 and 3).

$\mathrm{TNF}-\alpha$ is a key mediator in many experimental liver injury models $(20,21)$ and can exert differential functions through either of its cognate receptors, TNF-R1 and TNF-R2, which we found expressed in donor organs by RNA sequencing (RNAseq). TNF- $\alpha$ can downregulate platelet endothelial cell adhesion molecule 1 (PECAM-1), which may represent an important event in the sinusoidal transmigration of inflammatory cells later on $(22,23)$. TNF- $\alpha$ has also been shown to induce expression of CXCL8, one of the most potent chemoattractants and activators of neutrophils (24). Accordingly, CXCL8 was higher in blood samples from transplant IRI+ recipients prior to the operation, and it remained significantly increased at all intraoperative time points 

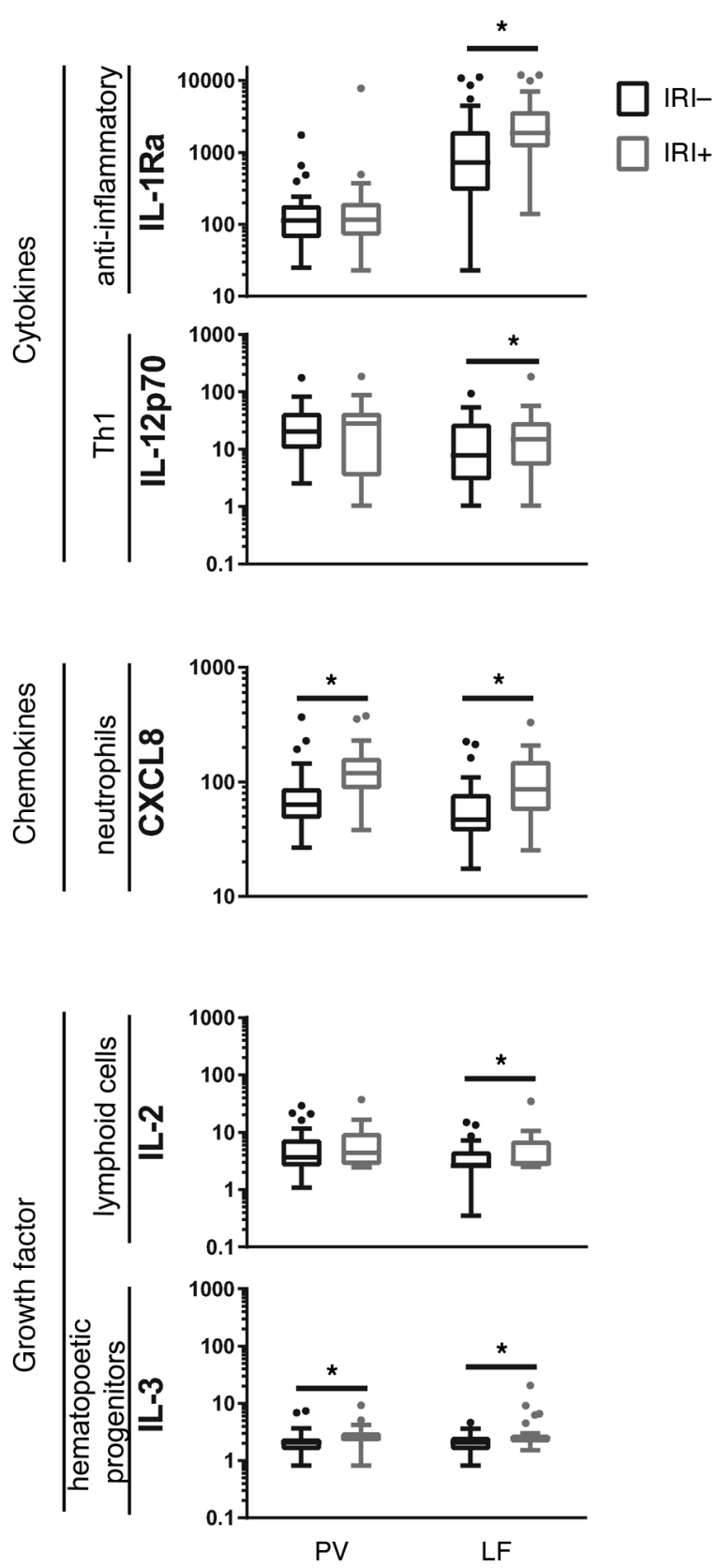

Figure 5. Donor and recipient cytokine, chemokine, and growth factors contribute differentially to OLT-IRI during reperfusion. Of 38 cytokines, chemokines, and growth factors tested by Luminex Multiplex assay, 5 were significantly higher in $\mathrm{IRI}+$ patients versus IRI- patients in patient portal vein blood either before (PV) or after (LF) it was flushed through the donor liver during reperfusion. Data are presented as Tukey box-and-whisker plots: whiskers are inner fences reaching 1.5 times the interquartile range and boxes represent the interquartile ranges, dots indicate outlying values, and lines represent median values for each time point. $n=53 ; 26 \mid \mathrm{RI}-$ and $27 \mathrm{IRI} \mid+{ }^{*} P<0.05$. The Wilcoxon rank-sum test was used for comparison between IRI+ and IRI- patient groups.

tested (PO and LF). Previous studies have indicated that prolonged cold ischemia time (>12 hours) leads to high CXCL8 plasma levels (25); however, our data indicate that IRI+ recipients have increased expression of this cytokine prior to the operation. In addition, IL-13, IL-2, and IL-1Ra were found increased $\mathrm{PO}$ and persisted across most time points tested in IRI+ recipients, implicating an involvement of adaptive immunity in the IRI process. The release of EGF is commonly reported as part of the injury-induced innate immune response, which can stimulate cellular growth, proliferation, and differentiation by binding to its receptor EGFR. Although we did not detect EGFR in biopsies of allograft tissue, aberrant EGF signaling through alternative pathways has been described, particularly in the context of cancer (26); therefore, it is possible that patient status prior to transplant could contribute to the actual function of this cytokine and its potential promotion of IRI.

At several time points after transplant, a rise in the levels of the cytokines IL-4, IL-13, and IL-17A as well as the lymphocyte growth factors IL-2 and IL-7 were observed in IRI+ patients, supporting our interpretation of a switch from an innate to an adaptive immune signature over time. Interestingly, IRI+ patients exhibited increased circulating IL-1a levels at W1 after transplant that were not accompanied by a significant increase in IL-1Ra, suggesting a resurgence of an acute inflammatory response. These findings suggest that a persistent chronic inflammatory response occurs, wherein both innate and adaptive immune responses continue to reactivate each other.

Importantly, we found expression of genes encoding most of the potential cognate receptors for the upregulated systemic cytokines, indicating that signaling could be initiated when recipient blood meets donor tissue. Exceptions to this were IL-3, IL-5, IL-7, and IL-12, which were increased at one or more time points in IRI+ patients but had no detectable receptor transcripts in biopsy tissues, indicating that the liver might not be a target organ for these cytokines. However, these cytokines might affect the immune system of the recipient, as their cognate receptors can be expressed on circulating immune cells. We did not observe significant changes in the level of cytokine, chemokine, and/or growth factor gene expression within the transcriptome data from IRI+ vs. IRI- biopsies, suggesting the donor liver released sequestered cytokines during IRI.

The liver is a prominent source of cytokines (11), and, as such, the hepatic extracellular matrix is a repository of cytokines, chemokines, and growth factors that can be released when required to be used by proximal cells, contributing to cellular programming $(27,28)$. In transplant, a unique situation occurs in which the cytokine profile of the donor allograft combines with that of the recipient, as cytokines produced in the donor allograft are likely to be flushed into the patient's circulating blood during reperfusion. It has been shown that donor-derived cytokines contribute to hemodynamic instability during reperfusion (29), which could affect IRI severity. To determine the contribution of the donor organ to cytokine release and patient IRI status, we directly compared blood samples obtained from the recipient's portal blood before or after reperfusing the donor organ as well as characterizing cytokine profiles of systemic blood samples obtained from recipients after transplant. CXCL8 and IL-3 increased in PV blood from IRI+ patients, similar to the systemic circulation. These two cytokines remained high in LF samples. However, 

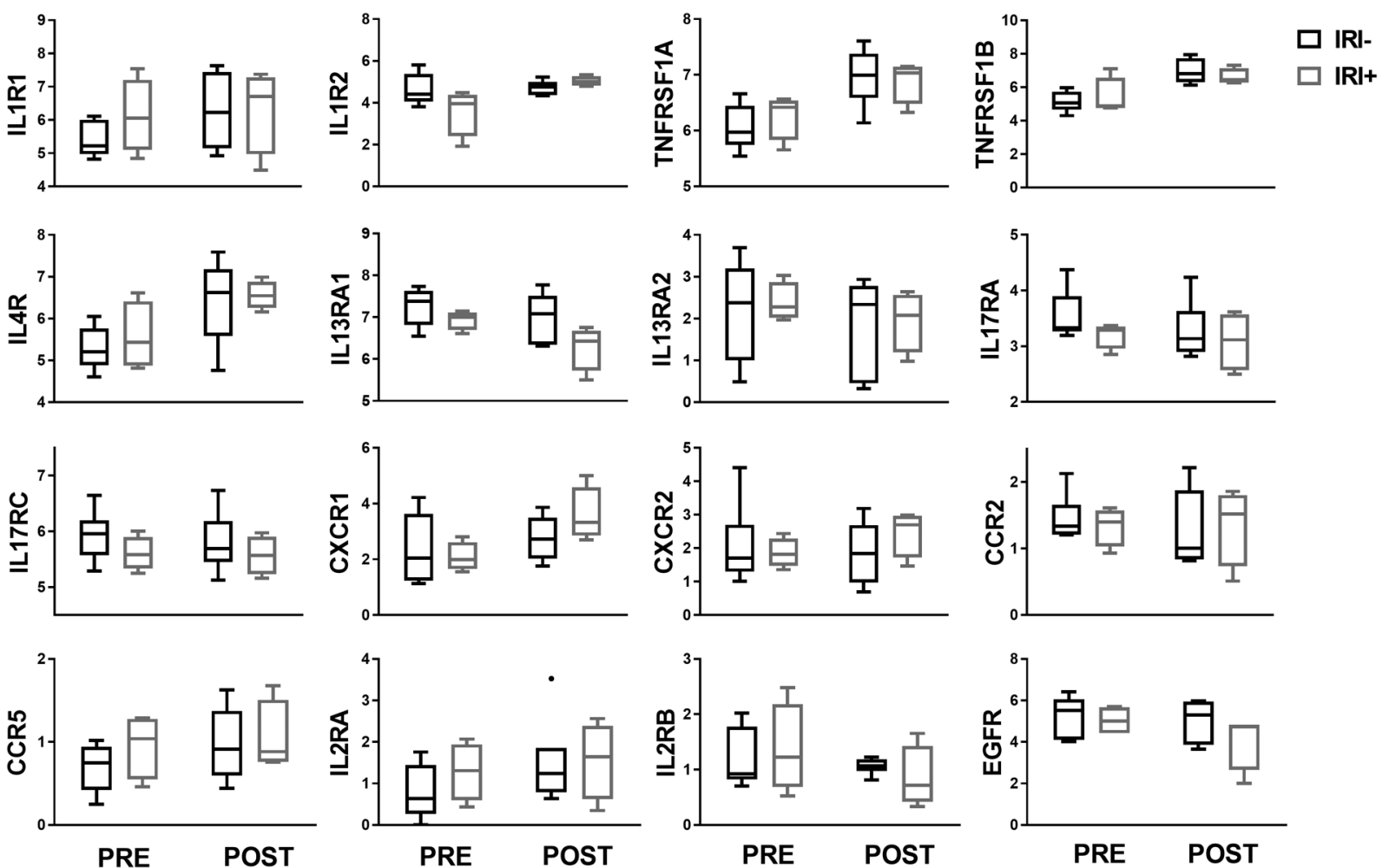

Figure 6. Transcripts of genes encoding cognate receptors for $\mathbf{1 0}$ of the $\mathbf{1 4}$ IRI-related cytokines are expressed in donor biopsies. Gene expression (transcripts per million; $\log _{2}$ TPM) in biopsies before and after reperfusion of IRI+ and IRI- OLT recipients by RNAseq. Data are presented as Tukey box-and-whisker plots: whiskers are inner fences reaching 1.5 times the interquartile range and boxes represent the interquartile ranges, dots indicate outlying values, and lines represent median values for each time point. $n=10 ; 6|R|$ - and $4|R|+$.

LF from IRI+ patients showed significant increases in IL-2, IL-12p70, and IL-1Ra that were not seen in PV. These results suggest that cytokines with a strong potential to shape the adaptive immune response (IL-2 and IL-12p70) come initially from the injured donor tissue. Additionally, high levels of IL-1Ra could contribute to the switch from innate to adaptive responses in IRI+ patients by dampening the innate component. In addition to secreted soluble IL-1Ra, there are two intracellular isoforms that comprise a reservoir of IL-1Ra that is only released upon cellular death, theoretically serving to limit the proinflammatory action of tissue damage (30). Importantly, we confirmed the expression of IL-1Ra and CXCL8 in donor biopsies by RNAseq.

Cytokines are pleiotropic and, as such, can exhibit redundant and/or opposing functions $(31,32)$. However, functional characterizations have typically resulted from animal studies in which a single cytokine is genetically or conditionally deleted, which may or may not represent what is occurring in human patients. Nevertheless, we found many cytokines related to IRI in human OLT at each of the time points tested, and studies conducted in various animal models have been instrumental in characterizing their key functional role(s) in mediating various aspects of IRI (33). For example, we found higher levels of TNF- $\alpha$ in IRI+ OLT recipients prior to surgery, and it has been shown that TNF- $\alpha$ is the central component of the proinflammatory cytokine cascade in liver IRI as well as a crucial effector of remote organ damage following IRI in a rat model (34). In a mouse model of IRI, it was further shown that TNF- $\alpha$ leads to the upregulation of ICAM-1, VCAM-1, and P-selectin, key adhesion molecules that are involved in the extravasation of inflammatory cells into the liver (35). Along these lines, animal models have pointed to the key involvement of several chemokines, such as CCL2, CXCL2, and CXCL10, in directing and regulating the accumulation of neutrophils, monocytes/macrophages, and T cells to the liver after IRI (33), and here, we report the increased expression of these and other chemokines as well as the specific time point that they become most important in human OLT-IRI. However, IFN- $\gamma$, IL-1 $\beta$, IL-6, and IL-10 have also been shown to be important regulators of IRI in 
Table 3. OLT patient outcomes in the first year after transplant

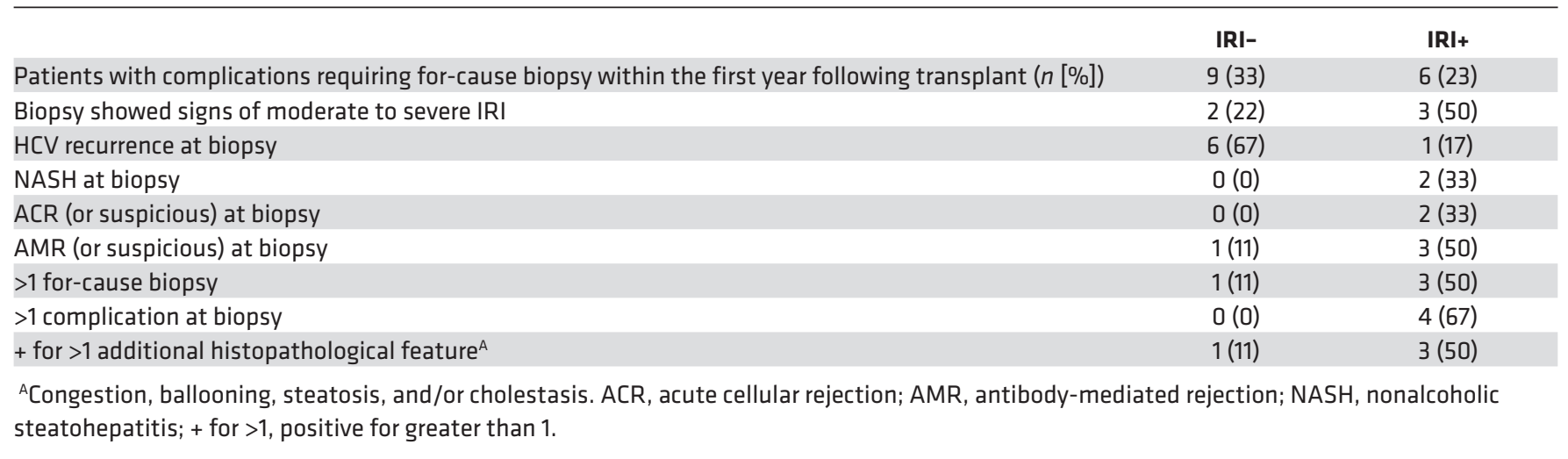

multiple studies using mouse and rat models, their exact roles are still debated (36-43), and we did not see any statistical difference in median values for these cytokines in our cohort of IRI+ and IRI- OLT patients. Therefore, although it is useful to categorize these individual soluble mediators of IRI on an individual basis by the location or functional involvement determined in highly controlled animal studies, future studies will be necessary to determine the significance of specific cytokine groups working either in concert or in a particular dynamic pattern. Toward this end, we have identified cytokine phenotypes in OLT patients based on a broader, less biased (albeit more complex) systems biology approach.

Features specific to human OLT-IRI are not well defined; thereby, classifications of patients as IRI- or IRI+ potentially conflict between different centers. Many studies use the Suzuki scoring system (12), or a center-specific modification, to evaluate biopsy samples taken just after the reperfusion event to determine extent of liver damage and/or one or more clinical tests to determine liver function following OLT. The Suzuki grading of hepatocellular injury was developed using a rat model of OLT-IRI (12) and evaluates the degree of sinusoidal congestion, hepatocyte necrosis, and vacuolization, with recommendation to include neutrophilic infiltration in the evaluation. However, this method does not consider additional features that have been reported in IRI+ humans, such as hepatocyte ballooning degeneration and cholestasis, and does not describe type of vacuolization, whether microvesicular or macrovesicular. In addition, no data are available on the correlation of the Suzuki score and clinical liver function tests. Based on our evaluation, we recommend a standardized scoring system that grades severity of IRI based on neutrophilic infiltration and hepatocyte necrosis but which also grades 4 other histopathological features commonly present in biopsies of human OLT recipients after reperfusion. The reperfusion of an organ that has been ischemic for long periods of time can result in sinusoidal congestion, mitochondrial swelling, disruption of liver sinusoidal endothelial cells, and the collapse of the space of Disse, resulting in impaired bile secretion and severe cholestasis. Cholestasis then leads to intrahepatic accumulation of bile acids and other toxic compounds, with progression of liver pathology. Its presence at such an early time point (2 hours after reperfusion), although it is likely not consequent to IRI, indicates poor donor organ quality and might predispose patients to more severe IRI as time progresses. Hepatocyte ballooning is a form of apoptosis and, together with cholestasis, represents microcirculatory disturbances. Finally, evaluating steatosis is becoming more important as nonalcoholic fatty liver disease and nonalcoholic steatohepatitis continue to increase in prevalence. Although some aspects of IRI are not expected to fully develop within 2 hours of reperfusion, such as cholestasis, a large-scale retrospective study analyzing patients undergoing liver transplantation over a 10-year period at a single center recently described the positive predictive value of the intraoperative biopsies as an independent determinant of graft loss (44). In evaluating the outcomes of our patient cohort within the first year following transplant (Table 3), we found that OLT recipients designated as IRI+ based on our scoring system were more likely to suffer complications requiring multiple "for-cause" biopsies; presented with more severe complications, such as ACR or AMR; and showed signs of more than one complication at each biopsy.

Here, we describe the results of 4 clinical liver function tests and their relevance to biopsy-proven IRI using the standard classification at our center. First, although severe cholestasis was not apparent on biopsies 2 hours after reperfusion, total bilirubin was significantly higher in IRI+ patients compared with 

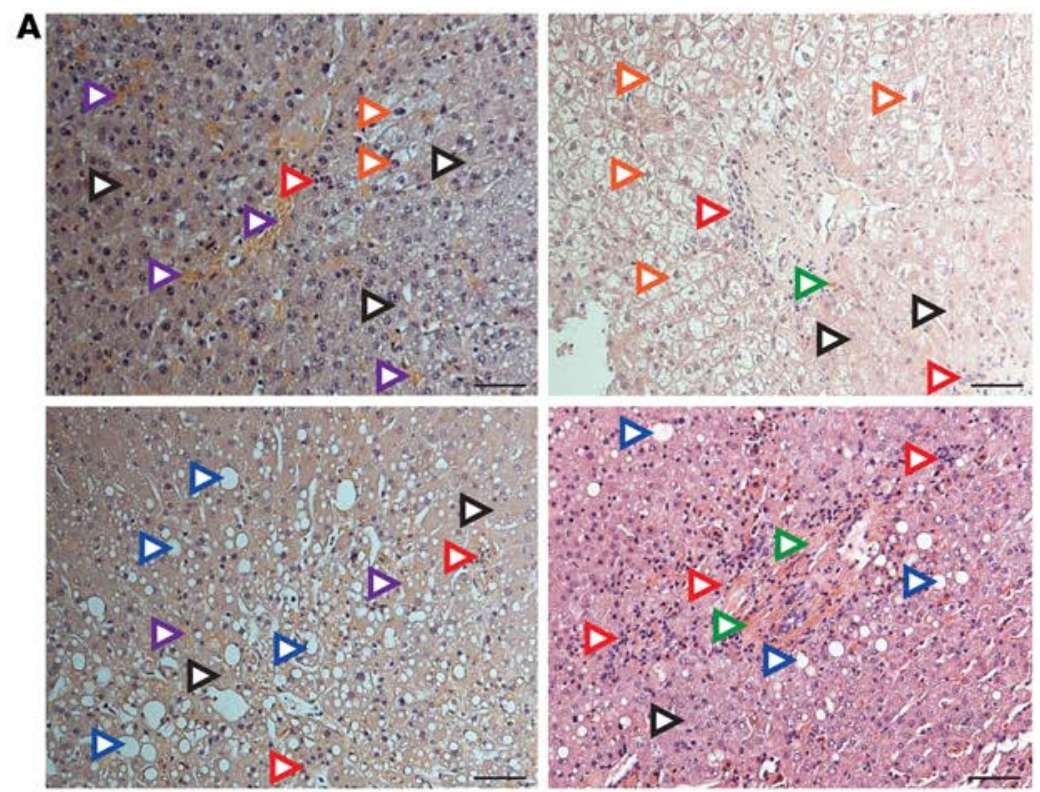

Symbol Legend:
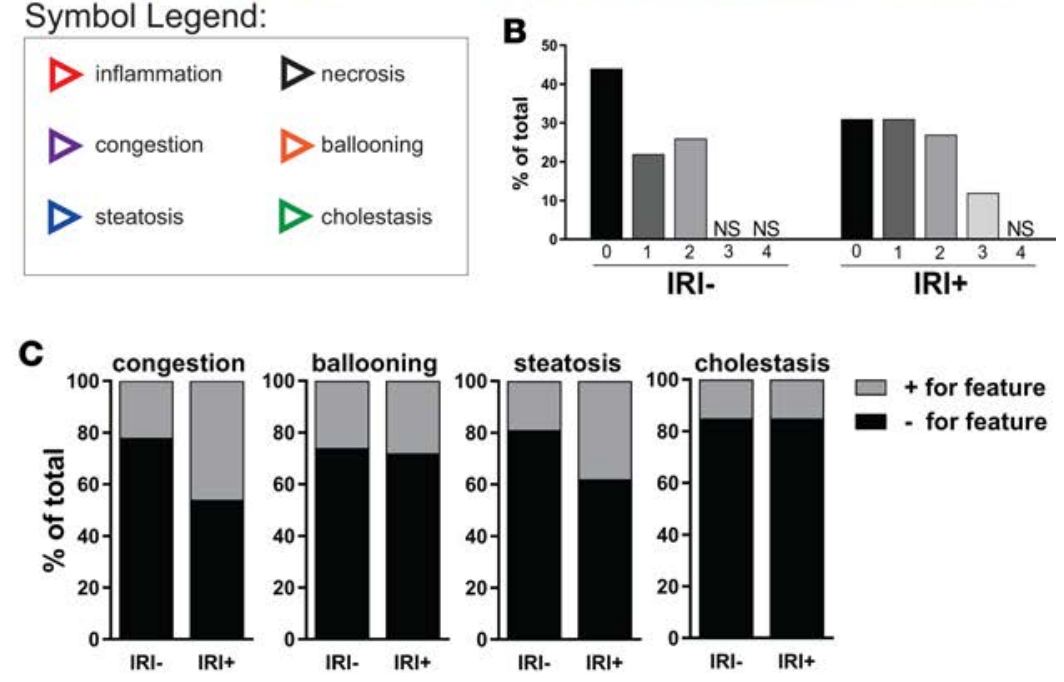

$\square+$ for feature - for feature

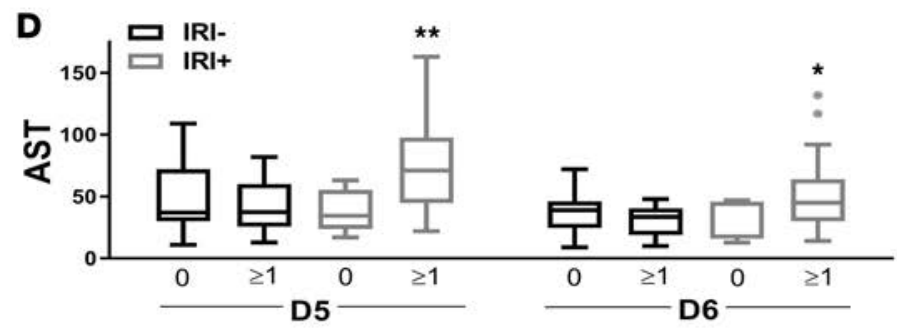

Figure 7. Histopathological features of liver IRI in human OLT. (A) Representative histology of IRI+ liver allograft biopsies after reperfusion illustrating the range of IRI seen on histology. IRI+ patients demonstrate marked inflammation (red arrowheads) and/or necrosis (black arrowheads) along with one or more of the following: sinusoidal congestion (purple arrowheads), hepatocellular ballooning degeneration (orange arrowheads), macrovesicular steatosis (blue arrowheads), and/ or cholestasis (green arrowheads). Scale bars: $50 \mu \mathrm{m}$. (B) Percentage of total IRI- or IRI+ patients that scored positive for 0 to 4 of the histopathological features of congestion, ballooning, steatosis, and/or cholestasis. NS, none scored for that data point. (C) Percentage of total IRI- or IRI+ patients either negative or positive for indicated histopathological features. (D) AST values for IRI- or IRI+ OLT recipients that presented with either 0 histopathological features or at least $1(\geq 1)$ additional histopathological feature(s) evaluated (congestion, ballooning, steatosis, and/or cholestasis). Data are presented as Tukey box-and-whisker plots: whiskers are inner fences reaching 1.5 times the interquartile range and boxes represent the interquartile ranges, dots indicate outlying values, and lines represent median values for each time point. $n=53 ; 26 \mid \mathrm{RI}-$ and $27|\mathrm{RI}|+.{ }^{*} P<0.05$, ${ }^{* *} P<0.01$. Statistical significance was determined using 1-way ANOVA with post-hoc Bonferroni for multiple comparisons and Bartlett's test for equal variances.

IRI- patients early after transplant; therefore, the detection of even a limited amount of cholestasis on an early biopsy is important for identifying at-risk patients. AST and ALT levels were high in both groups immediately after transplant and then dropped quickly in the following days. This is expected, as both enzymes are markers of cytolysis and this early transplant-related rise in liver enzymes is likely to cover subtler differences between IRI+ and IRI- patients. In line with this notion, we could pick up differences between the two patients groups only on days 5 and 6 after transplant, after this early rise had subsided. Finally, INR did not differ between IRI+ and IRI- patients, suggesting that liver biosynthetic function is not affected during the IRI process. Our data show that, in OLT patients, necrosis and inflammation, along with one or more of these additional features, correlated significantly with elevated AST levels in the first week after transplant, suggesting that these features might aggravate the IRI status of the patient. Additionally, a greater number of IRI+ patients who developed complications that required a "for-cause" biopsy within the first year after transplant had more than one of the additional histopathological features compared with IRI- patients that required a "for-cause" biopsy (50\% IRI+ vs. 11\% IRI-).

Overall, our data suggest that IRI might result from a deleterious combination of an IRI-prone donor-specific immune state coupled with an IRI-prone recipient-specific immune state. We envision the selected panel of innate and adaptive immune cytokines, chemokines, and growth factors identified in this study will serve as noninvasive predictors of IRI. Testing of the panel before transplant predicts recipient/ donor pairs at risk of IRI and can be used to improve the accuracy of donor selection and to guide application of targeted therapy to dampen recipient elevated cytokines and/or their cognate receptors in the donor 
liver. Furthermore, monitoring of these biomarkers early after transplant can be employed for patient management and assessing efficacy of therapy. Therefore, judicious decision-making regarding donor-recipient matching according to their particular predisposition to IRI might be a beneficial preventative strategy toward limiting IRI and thereby improving allograft outcome. OLT-IRI represents an intriguing convergence of the innate and adaptive immune responses from recipient and donor immune systems. Nevertheless, IRI develops when partial or complete obstructions of blood vessels occur with subsequent restoration of flow and is the most frequent pathology in the human body, when one includes myocardial ischemia, stroke, traumatic or hemorrhagic injury, and surgery, in addition to transplantation. Therefore, the immune phenotypes and pathways we identify in OLT-IRI are likely to be broadly applicable to IRI in all solid organ transplantation as well as in other IRI-related disease such as stroke and myocardial infarction.

\section{Methods}

Study design and sample collection. Adult primary OLT recipients were recruited between May 10, 2013, and April 6, 2015 (Table 2). Routine standard of care and immunosuppressive therapy was administered, as specified by UCLA liver transplant protocols. Study data were collected and managed using REDCap electronic data capture tools hosted at UCLA (45). AST, ALT, bilirubin, and INR analyses were performed as part of the standard of care. Donor organs were procured from both donation after brain death and donation after circulatory death donors with standardized techniques. Organs were perfused with and stored in cold University of Wisconsin solution (ViaSpan; Bristol-Meyers Squibb Pharma). Cold ischemia time was defined as the time from the perfusion of the donor with preservation solution to the removal of the liver from cold storage. Recipient venous blood was collected with acid-citrate-dextrose anticoagulant during two main phases relative to the transplant: PO and postoperative at D1, W1, and M1. Intraoperative portal blood was collected from the recipient PV prior to reperfusion (PV) and as it was first flushed through the vena cava of the donor liver during reperfusion (LF). Protocol Tru-Cut needle biopsies were taken from the left lobe intraoperatively after complete revascularization of the allograft ( 2 hours after reperfusion) prior to surgical closing of the abdomen. Extra care was taken to not sample the subcapsular region, as it is not representative of parenchymal liver tissue organization and can be affected by handling of the liver (46).

Liver histopathological IRI scoring. After reperfusion, biopsy samples were formalin fixed, paraffin embedded, and stained with H\&E, following a standardized protocol by the Translational Pathology Core Laboratory at UCLA. These were reviewed by a single transplant hepatopathologist and graded semiquantitatively for IRI. Two main pathologic findings were combined to make a global assessment of the severity of IRI: neutrophilic inflammation and hepatocyte necrosis (Table 1). Degree of neutrophilic infiltration was based on the presence of infiltrates as clusters within sinusoids. The degree to which hepatocytes are necrotic was based on the presence of necrotic hepatocytes as single cells or as clusters. Scores of $0-1$ represent no to minimal IRI (IRI-), and scores of 2-4 represent mild to severe IRI (IRI+). Additionally, we evaluated 4 histopathological features that were commonly found among biopsies of IRI+ patients. We based degree of sinusoidal congestion on presence by zone and number of lobules. Hepatocellular ballooning was defined as rare to prominent clusters of ballooning cells in one or more lobules. Macrovesicular steatosis was defined as large lipid droplets occupying over $50 \%$ of a cell and accompanied by indentation of the nucleus. Cytoplasmic vacuolization consisting of mostly small vacuoles with no nuclear displacement was not considered, because hepatocytes with only microvesicular steatosis are more likely to recover rather than complete the apoptosis process (47). Finally, we evaluated severity of canalicular or hepatocellular cholestasis based on presence in zone 3 of one or more lobules, with presence in zone 1 of any lobule being highest in severity. Images of H\&E-stained slides were acquired on a Nikon 90i microscope with NIS Elements software version 3.0 (Nikon).

Cytokine/chemokine Luminex assay. Plasma samples were prepared by centrifugation at $500 \mathrm{~g}$ for 12 minutes and stored at $-80^{\circ} \mathrm{C}$. Human 38-plex magnetic cytokine/chemokine kits (EMD Millipore, HCYTMAG-60K-PX38) were used per the manufacturer's instructions. Complete panel screened for the expression of the following: antiinflammatory cytokines: IL-1Ra and IL-10; general proinflammatory cytokines: IL-1 $\alpha$, IL-1 $\beta$, IL-6, IFN- $\alpha 2$, TNF/TNF- $\alpha$, TNF- $\beta /$ lymphotoxin $\alpha$ (LT- $\alpha$ ); lymphocyte-associated cytokine: soluble CD40 ligand (sCD40L); Th1/Th17-associated cytokine: IL-12p40; Th1-associated cytokines: IFN- $\gamma$ and IL-12/IL-12p70; Th2-associated cytokines: IL-4, IL-5, and IL-13; Th9-associated cytokine: IL-9; Th17-associated cytokine: IL-17A; neutrophil-associated chemokines: GRO/CXCL1 and IL-8/ CXCL8; eosinophil-associated chemokines: eotaxin/CCL11 and macrophage-derived chemokine (MDC/ CCL22); T cell/monocyte-associated chemokines: fractalkine/CX3CL1, IFN- $\gamma$-inducible protein-10 (IP- 
10/CXCL10), monocyte chemoattractant protein-1 (MCP-1/CCL2), and monocyte chemoattractant protein-3 (MCP-3/CCL7); leukocyte-associated chemokines: macrophage inflammatory protein-1 $\alpha$ (MIP-1 $\alpha /$ CCL3) and macrophage inflammatory protein-1 $\beta$ (MIP-1 $\beta$ /CCL4); lymphoid growth factors: IL-2, IL-7, and IL-15; myeloid growth factor: granulocyte-macrophage colony-stimulating factor (GM-CSF); hematopoietic progenitor growth factors: Fms-related tyrosine kinase 3 ligand (Flt-3L/CD135), granulocyte colony-stimulating factor (G-CSF), and IL-3; and other growth factors: EGF, FGF-2, TGF- $\alpha$, and VEGF. Fluorescence was quantified using a Luminex 200 instrument. Cytokine/chemokine concentrations were calculated using Milliplex Analyst software version 4.2 (EMD Millipore). Luminex assay and analysis were performed by the UCLA Immune Assessment Core.

Hierarchical clustering analysis. Analyte abundances irrespective of conditions were normalized using a robust variation of $\mathrm{Z}$-scaling that replaces the mean with the median and the SD with the median absolute deviation. Then, medians of scaled values for each group were color coded and plotted in heatmaps. Unsupervised hierarchical clustering was performed on rows and columns using Euclidean distance as the similarity measure with Ward's linkage.

RNAseq analysis. RNAseq testing was performed by the UCLA Clinical Microarray Core. Total RNA was first enriched for mRNA with the NEB NEBNext Poly(A) mRNA Magnetic isolation kit. The mRNA samples were then put on a IntegenX Apollo 324 system for automated library preparation using the IntegenX PrepX RNA-Seq Library reagent kit for Illumina. Library products were next enriched through PCR, and multiplexing barcodes were also added. Sequencing was performed on Illumina HiSeq 2000 for a single read 50 run. Data quality check was done on Illumina SAV. Demultiplexing was performed with Illumina CASAVA 1.8.2. Demultiplexed FASTQ files were checked with FASTQC. Reads were trimmed with Cutadapt to remove adapters and low-quality ends. Alignment to the hg38 human genome assembly was performed using STAR 2.5.1b (48). Unmapped reads, multi-mappers, and low-quality alignments were removed with Samtools. Reads mapping gene exons were counted using featureCounts (49). Counts were imported in $\mathrm{R}$ version 3.2.3 for analysis using the edgeR and ggplot2 packages (50). A negative binomial generalized log-linear model was fitted to the read counts for each gene. Statistical significance was determined using a likelihood ratio test, and $P$ values were adjusted according to Benjamini-Hochberg (50). Transcript abundances are expressed as transcripts per million (51), $\log _{2}$ scale. Data have been deposited in NCBI's Gene Expression Omnibus (GEO) and are accessible through GEO series accession number GSE87487.

Statistics. Wilcoxon nonparametric rank-sum test was used for comparisons between IRI+ and IRIpatient groups. For correlation between demographic data and IRI outcome, 2-tailed $t$ test was used for continuous variables and Fisher's exact test was used for categorical variables. A $P$ value less than 0.05 was considered significant.

Study approval. All studies described were reviewed and approved by the UCLA IRB (13-000143). Patients provided informed consent prior to their participation in the study.

\section{Author contributions}

RAS and AZ contributed equally to this work. RAS, AZ, MR, CRL, PR, AH, RWB, DWG, Y Zhai, JWKW, and EFR designed research studies. RAS, AZ, MR, CRL, ND, NH, and Y Zheng conducted experiments and/or acquired data. RAS, AZ, MR, CRL, ND, RS, and DWG analyzed data. RAS, MR, and EFR wrote the manuscript. AZ, CRL, BVN, PR, DWG, Y Zhai, and JWKW provided critical review of the manuscript.

\section{Acknowledgments}

This work was supported by the Ruth L. Kirschstein National Research Service Award (T32CA009120 to RAS) and Keck Foundation Award (986722 to JWKW and EFR). RS acknowledges support from a Quantitative \& Computational Biosciences (QCB) Collaboratory Postdoctoral Fellowship and the QCB Collaboratory community, directed by Matteo Pellegrini. We would like to thank Gregg Kunder and the liver transplant nurse team who go above and beyond to collect patient samples for this project. The authors hereby express their thanks for all of the organ donors and their families, for giving the gift of life and the gift of knowledge with their generous donation.

Address correspondence to: Elaine F. Reed, 1000 Veteran Ave., Los Angeles, California 90095 USA. Phone: 310.794.4943; E-mail: ereed@mednet.ucla.edu. 
1. Zhai Y, Busuttil RW, Kupiec-Weglinski JW. Liver ischemia and reperfusion injury: new insights into mechanisms of innate-adaptive immune-mediated tissue inflammation. Am J Transplant. 2011;11(8):1563-1569.

2. Zwacka RM, Zhang Y, Halldorson J, Schlossberg H, Dudus L, Engelhardt JF. CD4(+) T-lymphocytes mediate ischemia/reperfusion-induced inflammatory responses in mouse liver. J Clin Invest. 1997;100(2):279-289.

3. Shen X, et al. CD4 T cells promote tissue inflammation via CD40 signaling without de novo activation in a murine model of liver ischemia/reperfusion injury. Hepatology. 2009;50(5):1537-1546.

4. Shen XD, et al. Stat4 and Stat6 signaling in hepatic ischemia/reperfusion injury in mice: HO-1 dependence of Stat4 disruption-mediated cytoprotection. Hepatology. 2003;37(2):296-303.

5. Ji H, et al. Programmed death-1/B7-H1 negative costimulation protects mouse liver against ischemia and reperfusion injury. Hepatology. 2010;52(4):1380-1389.

6. Zhai Y, Petrowsky H, Hong JC, Busuttil RW, Kupiec-Weglinski JW. Ischaemia-reperfusion injury in liver transplantation--from bench to bedside. Nat Rev Gastroenterol Hepatol. 2013;10(2):79-89.

7. Hickman PE, Potter JM, Pesce AJ. Clinical chemistry and post-liver-transplant monitoring. Clin Chem. 1997;43(8 Pt 2):1546-1554.

8. Ramadori G, Armbrust T. Cytokines in the liver. Eur J Gastroenterol Hepatol. 2001;13(7):777-784.

9. Mackay IR. Hepatoimmunology: a perspective. Immunol Cell Biol. 2002;80(1):36-44.

10. Marrone G, Shah VH, Gracia-Sancho J. Sinusoidal communication in liver fibrosis and regeneration. J Hepatol. 2016;65(3):608-617.

11. Moshage H. Cytokines and the hepatic acute phase response. J Pathol. 1997;181(3):257-266.

12. Suzuki S, Toledo-Pereyra LH, Rodriguez FJ, Cejalvo D. Neutrophil infiltration as an important factor in liver ischemia and reperfusion injury. Modulating effects of FK506 and cyclosporine. Transplantation. 1993;55(6):1265-1272.

13. Okaya T, et al. Age-dependent responses to hepatic ischemia/reperfusion injury. Shock. 2005;24(5):421-427.

14. Yokoyama Y, Nagino M, Nimura Y. Which gender is better positioned in the process of liver surgery? Male or female? Surg Today. 2007;37(10):823-830.

15. Chung IS, et al. Incidence and predictors of post-reperfusion syndrome in living donor liver transplantation. Clin Transplant. 2012;26(4):539-543.

16. Siniscalchi A, et al. Hyperdynamic circulation in acute liver failure: reperfusion syndrome and outcome following liver transplantation. Transplant Proc. 2010;42(4):1197-1199.

17. Stahl JE, Kreke JE, Malek FA, Schaefer AJ, Vacanti J. Consequences of cold-ischemia time on primary nonfunction and patient and graft survival in liver transplantation: a meta-analysis. PLoS One. 2008;3(6):e2468.

18. Alvarez AM, Mukherjee D. Liver abnormalities in cardiac diseases and heart failure. Int J Angiol. 2011;20(3):135-142.

19. Veteläinen R, van Vliet AK, van Gulik TM. Severe steatosis increases hepatocellular injury and impairs liver regeneration in a rat model of partial hepatectomy. Ann Surg. 2007;245(1):44-50.

20. Schümann J, Tiegs G. Pathophysiological mechanisms of TNF during intoxication with natural or man-made toxins. Toxicology. 1999;138(2):103-126.

21. Bradham CA, Plümpe J, Manns MP, Brenner DA, Trautwein C. Mechanisms of hepatic toxicity. I. TNF-induced liver injury. Am J Physiol. 1998;275(3 Pt 1):G387-G392.

22. Neubauer K, Ritzel A, Saile B, Ramadori G. Decrease of platelet-endothelial cell adhesion molecule 1-gene-expression in inflammatory cells and in endothelial cells in the rat liver following $\mathrm{CCl}(4)$-administration and in vitro after treatment with TNFalpha. Immunol Lett. 2000;74(2):153-164.

23. Knittel T, et al. Expression and regulation of cell adhesion molecules by hepatic stellate cells (HSC) of rat liver: involvement of HSC in recruitment of inflammatory cells during hepatic tissue repair. Am J Pathol. 1999;154(1):153-167.

24. Osawa Y, et al. Tumor necrosis factor alpha-induced interleukin-8 production via NF-kappaB and phosphatidylinositol 3-kinase/Akt pathways inhibits cell apoptosis in human hepatocytes. Infect Immun. 2002;70(11):6294-6301.

25. Schmidt A, Tomasdottir H, Bengtsson A. Influence of cold ischemia time on complement activation, neopterin, and cytokine release in liver transplantation. Transplant Proc. 2004;36(9):2796-2798.

26. Amann J, et al. Aberrant epidermal growth factor receptor signaling and enhanced sensitivity to EGFR inhibitors in lung cancer. Cancer Res. 2005;65(1):226-235.

27. Wells RG. The role of matrix stiffness in regulating cell behavior. Hepatology. 2008;47(4):1394-1400.

28. March S, Hui EE, Underhill GH, Khetani S, Bhatia SN. Microenvironmental regulation of the sinusoidal endothelial cell phenotype in vitro. Hepatology. 2009;50(3):920-928.

29. Bezinover D, et al. Release of cytokines and hemodynamic instability during the reperfusion of a liver graft. Liver Transpl. 2011;17(3):324-330.

30. Garlanda C, Dinarello CA, Mantovani A. The interleukin-1 family: back to the future. Immunity. 2013;39(6):1003-1018

31. Paul WE. Pleiotropy and redundancy: T cell-derived lymphokines in the immune response. Cell. 1989;57(4):521-524.

32. Leonard WJ. The defective gene in X-linked severe combined immunodeficiency encodes a shared interleukin receptor subunit: implications for cytokine pleiotropy and redundancy. Curr Opin Immunol. 1994;6(4):631-635.

33. Abu-Amara M, Yang SY, Tapuria N, Fuller B, Davidson B, Seifalian A. Liver ischemia/reperfusion injury: processes in inflammatory networks--a review. Liver Transpl. 2010;16(9):1016-1032.

34. Peralta C, et al. Preconditioning protects against systemic disorders associated with hepatic ischemia-reperfusion through blockade of tumor necrosis factor-induced P-selectin up-regulation in the rat. Hepatology. 2001;33(1):100-113.

35. Rajesh M, et al. Cannabinoid-2 receptor agonist HU-308 protects against hepatic ischemia/reperfusion injury by attenuating oxidative stress, inflammatory response, and apoptosis. J Leukoc Biol. 2007;82(6):1382-1389.

36. Tsuchihashi S, et al. Vascular endothelial growth factor antagonist modulates leukocyte trafficking and protects mouse livers against ischemia/reperfusion injury. Am J Pathol. 2006;168(2):695-705.

37. Day YJ, Marshall MA, Huang L, McDuffie MJ, Okusa MD, Linden J. Protection from ischemic liver injury by activation 
of A2A adenosine receptors during reperfusion: inhibition of chemokine induction. Am J Physiol Gastrointest Liver Physiol. 2004;286(2):G285-G293.

38. Teoh N, Field J, Farrell G. Interleukin-6 is a key mediator of the hepatoprotective and pro-proliferative effects of ischaemic preconditioning in mice. J Hepatol. 2006;45(1):20-27.

39. Kato A, Gabay C, Okaya T, Lentsch AB. Specific role of interleukin-1 in hepatic neutrophil recruitment after ischemia/reperfusion. Am J Pathol. 2002;161(5):1797-1803.

40. Dinant S, Veteläinen RL, Florquin S, van Vliet AK, van Gulik TM. IL-10 attenuates hepatic I/R injury and promotes hepatocyte proliferation. J Surg Res. 2007;141(2):176-182.

41. Yagihashi A, et al. Downregulation of cytokine-induced neutrophil chemoattractants and reduction of reperfusion injury in liver allograft by interleukin-10. Transplant Proc. 2000;32(7):2302.

42. Welborn MB, Moldawer LL, Seeger JM, Minter RM, Huber TS. Role of endogenous interleukin-10 in local and distant organ injury after visceral ischemia-reperfusion. Shock. 2003;20(1):35-40.

43. Zhai Y, et al. Type I, but not type II, interferon is critical in liver injury induced after ischemia and reperfusion. Hepatology. 2008;47(1):199-206.

44. Ali JM, et al. Analysis of ischemia/reperfusion injury in time-zero biopsies predicts liver allograft outcomes. Liver Transpl. 2015;21(4):487-499.

45. Harris PA, Taylor R, Thielke R, Payne J, Gonzalez N, Conde JG. Research electronic data capture (REDCap)--a metadata-driven methodology and workflow process for providing translational research informatics support. J Biomed Inform. 2009;42(2):377-381.

46. van den Broek MA, et al. Liver manipulation during liver surgery in humans is associated with hepatocellular damage and hepatic inflammation. Liver Int. 2013;33(4):633-641.

47. Cazanave SC, Gores GJ. Mechanisms and clinical implications of hepatocyte lipoapoptosis. Clin Lipidol. 2010;5(1):71-85.

48. Dobin A, et al. STAR: ultrafast universal RNA-seq aligner. Bioinformatics. 2013;29(1):15-21.

49. Liao Y, Smyth GK, Shi W. featureCounts: an efficient general purpose program for assigning sequence reads to genomic features. Bioinformatics. 2014;30(7):923-930

50. Robinson MD, McCarthy DJ, Smyth GK. edgeR: a Bioconductor package for differential expression analysis of digital gene expression data. Bioinformatics. 2010;26(1):139-140.

51. Li B, Ruotti V, Stewart RM, Thomson JA, Dewey CN. RNA-Seq gene expression estimation with read mapping uncertainty. Bioinformatics. 2010;26(4):493-500. 